\title{
Anticuerpos monoclonales en la evaluación de enzimas del ribete estriado yeyunal de lactantes con diarrea persistente
}

\author{
Magdalena Araya Q.'; Julio Espinoza M.'; Francisco Alliende G.'; M. Jesús Rebollo G. ${ }^{2}$; \\ Oscar Brunser T. ; Francisco Barrera Q. ${ }^{2} ;$ Buford Nichols ${ }^{3}$
}

\author{
Evaluation of microvilli enzimes by monoclonal antibodies \\ in infants with longstanding diarrhea
}

\begin{abstract}
Express on si smol inesline microvilli enzimes lac ase, sucrase-isomaliase, moliose ond aminopeptidase by monoclonal aribodies was studied in biopsies of yeyuna muceso fror eight intarts lage 2 to 12 moritist and two boddlers laged 16 and 33 months, wh persisten diarnea. Results were comparea with clinical findings, mucosal morphocgy ilight microscopyl, disocchoridase activities |Dah.quisti, and mistochemıcol expression of loctase. Amınopeptidase was expressed jy monocionol anibodies in all potents, both in crypts and villi. Lachase expession by monctonal ontibodies in villi wos in agreement with rest ts of histochemical and Catilquist rrethods while ir only two $\mathrm{c}^{-}$itdrens he corespanding enzimes became expressed by monocleral antibodies in crypls. Su-roseisomaliase expression by monoclonict artibodies in villi tended to pe more frequent in patien's where mucosol morphology was best presenved. excep: fer one cose who had a primary enzime deficiency, even though they were defectec in the crypls of atl but two cases that also showed ne expression in their v l.i. Evaluation of microvilli enzimes by moncolonal antibody expression in paients with secondary damage to the srioll intestine may be useul, becasse it may erhonce the urderslanding of impairment and reouir mecranisms and help to es'imote oregrosis of patien's with lorgstanding diartheo.
\end{abstract}

iKey words: diarneo pessistent, infant, i-testinaf mucosa, miciovilli anribodies monacioncl. aninopeptideses, disacchoridases. !

El diagnóstico y tratamiento de pacientes con diarrea persistente grave han mejorado considerablemente en los últimos años, sin embargo, ella continúa siendo responsable de parte importante de la letalidad por enfermedad diarteica, causa desnutrición severa y su tratamiento tiene alto coste cn alimentaciones especiales y largas hospitalizaciones ${ }^{1-4}$.

Un factor frecuente de diarrea persistente es la intolerancia a hidratos de carbono, incluyendo lactosa, sacarosa y almidones. La deficiencia enzimática puede durar períodos prolongados, favoreciendo la pérdida de nutrientes, aparición o agravamiento de la desnutrición y pululación

I. Unidad de Gastrocnterologia, lnstituto de Nutrición y Tecnología de los Alimentos (INTA), Universidad de Chile.

2. Servicio de Pediatría. Hospital San Borja-Arriarán.

3. Children Nutrition Research Center (CNRC). Texas, Houston, USA.

Financiado parcialınente por FONDECYT 194-033.3. bacteriana. Estas intolerancias se evaluan principalmente mediante medición del pH y sustancias reductoras en deposiciones, estimación de la actividad enzimática en la mucosa intestinal, curvas de tolerancia al hidrato de carbono que se supone ofensor y pruebas clínicas de privación dietética de éste. Desde que se dispone de anticuerpos nonoclonales (AcM) para las enzimas del ribete estriado, se ha abierto una nueva forma de evaluar las lesiones de la mucosa intestimal, que podría facilitar la comprensión de los mecanismos de daño y reparación de dichas enzimas. Con ellos y otros métodos de estudio sobre la biología molecular de las enzimas se ha podido determinar que las fallas congénitas de disacaridasas, especialmente sacarasa. pueden obedecer a errores en la sintesis, inserción o activación de la enzima en la membrana. Sin embargo, la información sobre los cambios que ocurren en casos no congénitos es muy esca$\mathrm{sa}^{5-9}$. El propósito de esta experiencia fue evaluar la información obtenida con el método de 
identificación de enzimas del ribets estriado mediante anticuerpos monoclonales, ел comparación con otros más corrientes.

\section{Material y Métodos}

Se estudiaron los diez lactantes con diarcea persistente de intensidad muderadia a grave en que se efectuó una biopsia de yeyuno somo parti de su svaluación durante el año 1993. La muestra de mucosa ysyanal ste obuvo en el ángralo de Treitz mediante una cápsula pediátrica de Crosby-Kugler de doble puerta (Quinton Instruments, Sealle, WA). Uno de los trozos se dividió en dos: una de das mitades se fijó ca reactivo Bonin e incluyo en parafina, para cortarla en strics, teñírla con he rnatoxilina-eosina $y$ estudiarla por microseopia de luz: los resultados morroIógicos st tradujeron a una escala de 1 a 4 . en gut uno corresponde a la estructura normal y cuatro al aplanamiento total de la superficic, en perdida de veliosidades. hipertrofia de las criptas e intensa itofiltración celular de lo lamina propia ("Inucosa plana") $\left.\right|^{10}$. La otra nuitad inceluyó en Tissuc-Tek OCT Compound (Elkhirt. IN). se congeló rápidamente en nitlogenes líquido y se mantuvo a $-70^{\circ} \mathrm{C}$ basta al momento on quc se obluvieron cortes de $5^{\circ}$ con un crióstato a $-20^{\circ} \mathrm{C}$, que fueron fijados sin actoma fría $y$ secatios a temperatura ambicnte: los cortes se incubaron con anticuerpos monoclonales contra lactasa, satarasa. maltasa y aлtinopepudasa, provistos por uno de los ututores (B. N.). El procedimiento usado ha sido descrito" $y$, en rtsumen, consistió en una técrica de emparedado en ques sta bloquea la tincion isespecifica con $0.2 \%$ de stroalbunina bovina, se incuba el corte con el anticuerpo monoclonal correspondienle y luego se réaliza una tumión de contraste con a7al de Evans. Como controles posilivos se usaron corles de biopsias de tejido sano oblenidass de pacicntes que requiritron un procedimiento gairúrgico con apertura o resección del intestino delgado. Otros cortes a los que no se agregó el anticuepo sirveron como controles negalivos. Los resultados se expresaron como presencia o ausen. cia de produsto de reación, de acterdo con el porcentaje de la superficie epilelial que mostraba reacción positiva. para lo cual se construyo una excala de I a 4 . donde I indica 67 a $100 \%$ : 2 de 34 a $66 \%, 3$ de 1 a $33 \%$ y 4 de $0 \%$. respectivamente, de la reacción obsertula en tejidos de sujetos normales. En las muestras de mucosa donde la re. acción sobre la supetficie epilejial no esa comtiuua. la distribución se describió como parcelar. El corte adyacenle a aquel que se incubaríu con anlituerpos motoclonales an. tilactusa se hizo de $10 \mu$ de espesor y fue empleado en la determinación histoguímical de lactasa, con medio de incubación preparado de acuerdo al mécodo de Lojida $y$ Kranll': sc incubos por 20 mintosios a $37^{\circ} \mathrm{C}$. luego fue lavado dos veces en clorure de sollio al $0.85 \%$, fijado en formaldehido al 4 \% lavado otras dos veces en agua deionizada. montado en glicerol con solteition salina-tampón tosfato ( 1 : 1. volutmen a volumen) y evaluado con microscopio de luz, definiendo la intensidau del color obtenido en una escala de 1 a 4 , semejante a la descrital para la eviluación de los anticuerpos monoclunales.

En olro trozo de la biopsia se midió la actividad de lactasia, sacarasa-isomailusa y maltasas con el método de
Dahdavis ${ }^{12}$. Se definieron como normales actividades mayores o igualcs a 10 UI de laclasa 40 U1 de sacurasaisomaltasa y on U de natlasi. Lon resultados se expresaron en escala de I a 5 . asignando calificación I al cquivaleate a $100 \%$ det promedio normal de actividad: 2 entre 67 y $99 \%$; 3 entre 34 y $66 \%: 4$ entre 1 y $33 \%$ y 5 a atsisucial de actividad enzinatica.

En todos los niños se realizaron, adentás. estudios etiológicos en busca de enteropatógenos backerianos, parasirarios y rotavirus, y los exánenes considecados mecesarios para su correcto manejo clínico.

\section{Resultados}

Las características clínicas más relevantes de los pacientes se exponen en la tabla 1. En el caso 1 el diagnóstico de egreso fue hetcrozigoto de deficiencia congénta de sacarasa-isomaltasa: en 3 años de seguimiento ha cvolucionado con alzas y bajas de los niveles de dicha enzima, fluctuando entre menos de $50 \%$ de lo normal a no delectable. caso este último en que se hacía muy sensible a pequeñas cantidades de sacarosa cn la dicta. El paciente 5 fue el único en que se aislatron agentes etiológicos (Cryptosporiditm sp. en una ocasión y Escherichia coli enteropatógeno en otra): es también cl único en que la microscopía de luz mostró daño moderado (grado 3), de tipo agudo, y las disacaridasas tuvieron valores colre 1 y 2 en todas las formas de medición. En el caso 10 sc sospechó inicialmentc intolerancia a proteína de leche de vaca. pero los csiudios dieron resultado negativo, excepto la IyA secretoria en saliva, que no era detectable. En los otros dos casos en que se planteó intolerancia a proteína de leche de vaca (pacientes 4 y 7 ) las manifestaciones clínicas eran muy sugerentes de esle diagnóstico y desaparecieron completamente cuando se suspendieron la lcche y productos lácteos de la dieta, pero no fue posible seguirlos. El caso 6 cra un niño con diarrea persistente grave, relractaria al tratamiento médico, que requirió alimentación parenteral casi permanentemente $y$ finalmente fallecio alrededor de los dos años, con desnutricion severa.

La evaluación de la mucosa intestinal desde e] punto de vista morlologico, histoquímico, por inmunofluorescencia y de la medición de la actividad enzimática aparecen en la tabla 2 . En ella se incluyen los resultados obtenidos en las vcllosidades con anticuerpos monoclonales. En las cliptas sólo se obtuvieron resultados positi- 


\section{Tabla I}

Características y diagnósticos de ingreso de pacientes con diarrea persistente

\begin{tabular}{|c|c|c|c|c|c|}
\hline$n$ & $\begin{array}{c}\text { Edad } \\
\text { (meses) }\end{array}$ & Sexo & $\begin{array}{l}\text { Diagulóstico } \\
\text { al ingreso }\end{array}$ & $\begin{array}{l}\text { Pesolddad } \\
\text { ( } \% \text { NCHS })\end{array}$ & Alimentación" \\
\hline 1 & 10 & Mase & DN/IHC & $60.0 \%$ & Pollo y verduras \\
\hline 2 & 10 & Fim & DNIIHC & $48,5 \%$ & Dicta modular \\
\hline 3 & 2 & Masc & DN/IHC & $75,4 \%$ & Al $110^{R}$ \\
\hline 4 & $1 i$ & Masc & IPLV & $92,9 \%$ & Prosobee ${ }^{\mathrm{k}}$ \\
\hline 5 & 8 & Fem & JNN/Cry/EPEC & $76,0 \%$ & Pollo y verduras \\
\hline 6 & 12 & Masc & DN/DP & $56.2 \%$ & Pregestimil ${ }^{R}$ \\
\hline 7 & 10 & Fern & DNIIPLV & $42.7 \%$ & $A D N^{R}$ \\
\hline 8 & 16 & Fem & $\mathrm{EC}$ & $76.9 \%$ & S/laclosa \\
\hline 9 & 4 & Masc & $\mathrm{IHC}$ & $99.3 \%$ & $A D N^{k}$ \\
\hline $\mathrm{j} 0$ & 33 & Mase & IPLV & $95,1 \%$ & S/leche de vaca \\
\hline
\end{tabular}

IHC: intolerancia a hidratos de carbono; DN: desnutrición: IPLV: intolerancia a proteina de leche de vaca: Cry: Crytosporridhum; EPEC: Fscherichia coli enteropatogena: DP: diartea persistente; EC: enfermedad celtaca. S/sin. Peso/Edad: porcentaje del percentil 50 de las tablas NCHS.

* Al momento de la biopsía

\section{Tabla 2}

Estudio morfológico y enzimático en biopsias yeyunales de pacienles con diarea persistente

\begin{tabular}{|c|c|c|c|c|c|c|c|c|c|c|}
\hline \multirow[t]{2}{*}{$\mathbf{n}$} & \multirow{2}{*}{$\begin{array}{l}\text { Diagnóstico } \\
\text { al egreso }\end{array}$} & \multirow[t]{2}{*}{ ML } & \multirow[t]{2}{*}{ HL } & \multicolumn{4}{|c|}{ Ac. monoclnnales } & \multicolumn{3}{|c|}{ Dablqrist } \\
\hline & & & & I. & $\mathbf{S}$ & MI & $\mathbf{A}$ & $\mathbf{L}$ & $\mathbf{s}$ & $\mathbf{M}$ \\
\hline 1 & ICS/DN & 2 & 3 & 3 & 4 & 3 & 3 & 1 & 3 & 2 \\
\hline 2 & DP/DN/IHC & $1-2$ & 1 & 1 & 4 & $i$ & I & $i$ & 5 & 2 \\
\hline 3 & DP/DN/IHC & $2-3$ & 1 & 1 & I & 1 & 2 & 1 & 2 & I \\
\hline 4 & IPLV/DN & $2 \cdot 3$ & 1 & 2 & I & 3 & I & I & 2 & I \\
\hline 5 & EPEC/Cry/DN & 3 & 1 & 1 & 1 & 1 & 2 & i & 2 & 1 \\
\hline 6 & DRT/DN/IL & $2-3$ & 4 & 3 & 2 & 3 & J & 3 & 3 & 3 \\
\hline 7 & [PLV/]L & 4 & 4 & 4 & 1 & 4 & 1 & 3 & 3 & 2 \\
\hline 8 & $\mathrm{EC} / \mathrm{IL}$ & 4 & 4 & 3 & 1 & 2 & i & 3 & 3 & 2 \\
\hline 9 & DP/DN/IHC & 2 & 1 & I & $i$ & i & i & I & i & 1 \\
\hline 10 & Del IgAs & 1 & 1 & I & I & i & i & 1 & ] & I \\
\hline
\end{tabular}

ICS: intolerancin congénita a la sacalosa; DN: desnutrición; IHC: intolerancia a hidratos de carbono; DP: diartea persistentc; IPLV: intolerancia a proteina de leche de vaca: EPEC: Escherictuet coli tenteropatógena: Cry: cryptosporidiosis: IL: intolerancia a la lictosa: DRT: diafrea refractaria al tratamienlo; EC: enlermedacl celiaca; Def IgAs: deficiencia de IgA sccretoria; ML: microscopia de tuz, 1 a 4; HL: histoquitnica de letelasa: Ac: anticuerpos: $1=66-100 \%$. $2=34$ $66 \%, 3=1-3.3 \%, 4=0 ;$ Metodo de Dahlquist: $]=10.0 \% .2=67-99 \%, 3=34-66 \%, 4=1-33 \%, 5=0$. L: lactasa; $\mathrm{S}$; sacarasa: M: miltasa: A: aminopeptidasa.

vos a histoquímica y anticuerpos monoclonales para lactasa en los pacientes 3 y 9 , en que la reacción correspondió al valor 3 en la escala de 1 a 4; los resultados con anticuerpos monoclonales para sacarasa-isomaltasa fueron positivos en las criptas en todos los casos menos los pacicntes 1 y 2 , en los que tampoco se observó expresión en las vellosidades. Los anticuerpos monoclonales contra maltasa dieron resultados positivos de forma parcelar, en algunas criptas, de los pacientes 3,9 y 10 con calificación 3. La aminopeptidasa fue positiva, en tasas normales, en todos los casos, tanto en las vellosidades como en las criptas. 


\section{Comentario}

Los pacientes que se comentan sufrían diarea prolongada y daño morfológico del intestino delgado de distinta magnitud, de lipo secundario. En tres no se hizo diagnóstico etiológico. y siete de diez sufrían desnutrición grave. De los bien nutridos, uno estaba en dieta sin leche de vaca desde hacia un mes y mostraba franca recuperación clínica y nutricional; en otro las manifestaciones hacían sospechar una intolerancia a sacarosa-isomaltosa que luego no se confirmó y en el tercero la enfermedad nunca afectó el estado nutricional durante toda la evolución.

Cuando la actividad de lactasa era baja con el método de Dahlquist, la reacción con el anticuerpo monoclonal correspondiente era también escasa o nula. Salvo en el paciente 1 , cuya actividad de lactasa era normal con el método de Dahlquist, mientras -como en los demás- la reacción con anticuerpos monoclonales era baja y también era mayor la actividad sacarásica con el primer método que con el segundo; en los casos restantes los anticuerpos monoclonales resultaron ser más sensibles para delectar sacarasa que cl mélodo enzimático. Podría pensarse que esto es lógico, pues estos anticuerpos monoclonales pesquisan todas las moléculas de enzina producidas, aunque no sean activas, ya que el epitope no está relacionado al sitio activo. La reacción con anticuerpos monoclonales para aminopeptidasa, normal o ligeramente disminuida en todos los casos salvo el paciente 1 , corresponde con lo observado desde el punto de vista clínico, pues a pesar de la seriedad de la enfermedad ringuno de los pacientes tenía problemas para manejar las proteínas que se les suministraban.

El paciente 1 mostró un comportaniento distinto al de los demás casos estudiados; mientras la arquitectura de su mucosa intestinal presentaba daño leve a moderado, no específico, los dos métodos utilizados para demostrar sacarasaisomaltasa en el ribcte estriado dieron resultados profundamente alterados. Tomando en cuenta la información publicada acerca de casos congénilos de deliciencia de esta enzima ${ }^{5-5}$, estos resultados podrían ser explicados postulando que cl paciente tenga una información genética alterada y que construya moléculas enzimáticas defectuosas, no pesquisables por el eptope que liga el anticuerpo monoclonal correspondiente, pero que no obstante tendrían intacta la región del sitio activo. Empleando juegos de doce antjcuetpos monoclonales contra sacarasa-isomaltasa de scres humanos adultos. se ha demostrado que las células de las vellosidades expresan una enzima distinta que la de las criptas, pero ambas tendrian un precursor común y su expresión dependería del estado de maduración de los enterocitos de donde provienen ${ }^{13}$. Con el anticuerpo monoclonal utilizado por nosotros no se detecto sacarasa-isomaltasa en vellosidades y criptas. Los resultados de este estudio difieren con otros obtenidos en niños desnutridos, con diarrea crónica, en los que todos los pacientes tenían expresión de sacarasa-isomaltasa en vellosidades y criptas, independientemente del grado de daño morfológico que presentaran".

Los anticuerpos monoclonales para enzimas del ribetc estriado aportan información novedosa acerca del comportamiento de éstas. Su utilización en condiciones clínicas que representen modelos de daño secundario permitirá entender mejor los mecanismos de la lesión y reparación de la mucosa intestinal y, eventualmente, pueden servir para estimar cl pronóstico de la atección intestinal.

\section{Resumen}

En las biopsias de mucosa yeyunal de diez pacientes con diarrea persistente se extudió la expresión de las enzimas lactasa. sacarasa-isomaltasa. maltasa y aminopeptidasa, tlel ribete estriado, mediante anticuerpos monoclonales y los resultados se contrastaron con los síntomas y signos clínicos, morfológicos (microscopía de luz). acrividad disacaridásica (Dablquist) y la expresión de lactasa por un método histoquímico. Se obtuvo expresión de aminopeptidasa en criptas y vellosidades de todos los niños, en criptas y vellosidades, mediante los anticuerpos correspondientes. La expresión por anticuerpos. histoquímica y actividad enzimática (Dahlqvist) fueron concordantes para la expresión de lactasa en las vellosidades, mientras en las criptas se registro positividad sólo en dos casos. En las veliosidades los anticuerpos monoclonales tendieron a producir más reacciones positivas para sacarasa-isomaltasa en los casos con menos daño morfológico, excepto en uno de deficiencia primaria; en las criptas el resultado fue posi- 
livo en todos, menos dos pacientes, en los que tampoco hubo positividad en las vellosidades. Los anticuerpos monoclonales pueden aportar información útil para crtender mejor los mecanismos de daño y reparación de las enzimas del ribete estriado y estimar el pronostico de la lesión.

(Palabras clave: diarrea persistente, Jactante, mucosa intestinal, microvellosidades, anticuerpos monoclonales, aminopeptidasas, disacaridasas.)

\section{Referencias}

1. Avery GB, Villaviencio $O$, Lilly J, Randolph $J$; Intractabie diarrhea in early infancy. Pediatrics 1968: 41: 712-722.

2. Lebenstal E: Intraciable diarthen of infancy. Texthook of gastroenterology and nutrition in infancy. Segunda edición. NY Raven Press 1989: 1077-1091.

3. Girautr D. Goulet $O$. Le Deist $F$, et al: Intractuble infant diarthea associated with phenotypic ahuornjalities and immunodeficiency. J Pediatr 1994; 125: 36-42.

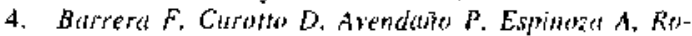
mery $G$, Escobom S: Diarrea refractaria. Rev Chil Pediatr 1989; 55: 316-320.

5. Hauri HP. Quaroni A. Liselhorhe KJ: Monoclonal antibodies to sucrase-isomaltase: prohes for the study of postnatal development and biogenesis of the intesti- nal microvillus membrane. Proc Nall Acad Sci USA 1980: 77: 6529-6633.

6. Quctroni A. Ssselbacher $K J$ : Study of cell differentation with monoclonal antibodies to intestinal cell surface cormponents. Dev Biol 1985; 11 1:267-279.

7. Hany HP, Rorh J. Sterchi E. Lenze M: Transport to cell surface of intestinal sucrase-isomaltase is blocked in the Golgi apparatus in a patient with congcnital sucrase-isomaltase deficjency. Proc Natl Acad Sci USA 1985; 82: 4423-4427.

8. Nush H. Sterchi $E$, Roth J, Hawri $H P$ : Congenital suctase-isomaltase deficiency in man. Evidence for diflerent mutations interfering with the intracellular transport of sucrase-isomaltase. Experientia 1988: 44: 64 (abstract).

9. Nichols B. Curraze $F$. Nichols $V$ et al Mosaic expression of brush border enzymes in infants with chronic diarthea and malnutrition. J Pediatr Gastroenterol Nutr 1992; 14: 371-387.

10. Buker 5: Geographical variations in the morphology of the small intestinal mucosa in apparently healthy individuals. Pathol Microbioi 1973; 39: 222-2.34.

11. Lrjdte $Z$. Kraml $J$ : lndigogenic methods for gluizosides. [II An improved method with-4-Cl-5-Br3-Indoly]-B-[D- Fucoside and its application in studies of enzmes in the intestine. kidney and other tissues. Hislochemmie 1971: 25: 195-207.

12. Dothquist A: Methods for assay of intestinal disaccharidases. Anal Biochem 1964; 7: 18-25.

13. Becutien J. Nichols B. Qusuromi A: Postranstational regutation of sucrase-isomalose expression in intestinal crypt and villus eel]s. J Biol Chem 1989; 264: 2(00)-2011.

\section{AVISO A LOS AUTORES}

Con el objeto de dar prioridad a los trabajos de investigación, en vista de las limitaciones de espacio de la Revista Chilena de Pediatría, el Comité Editorial ha acordado restringir la impresión de casos clínicos a un máximo de dos por cada número. 